\title{
Tradução e adaptação cultural para o português do Brasil do Children's Physical Activity Questionnaire (C-PAQ)*
}

\section{Translation and Cultural Adaptation to Brazilian Portuguese of the Children's Physical Activity Questionnaire (C-PAQ)}

\author{
Fernando Leite Miranda ${ }^{1}$ Carlos Henrique Fernandes ${ }^{1,2(0)}$ Lia Miyamoto Meirelles ${ }^{1}$ (으 \\ Flavio Faloppa ${ }^{1,2(0)}$ Benno Ejnisman ${ }^{1,2}{ }^{-0}$ Moises Cohen ${ }^{1,2(0)}$
}

\footnotetext{
1 Programa de Pós-graduação em Ciências da Saúde Aplicada ao Esporte e à Atividade Física, Departamento de Ortopedia e Traumatologia, Escola Paulista de Medicina, Universidade Federal de São Paulo (Unifesp), São Paulo, SP, Brasil

2 Departamento de Ortopedia e Traumatologia, Escola Paulista de Medicina, Universidade Federal de São Paulo (Unifesp), São Paulo, SP, Brasil
}

Rev Bras Ortop 2021;56(5):574-578.
Endereço para correspondência Carlos Henrique Fernandes, MD, PhD, Avenida Leôncio de Magalhães, 1.021, São Paulo, 02042-011, SP, Brasil (e-mail: carloshandsurgery@gmail.com).

\begin{abstract}
Resumo
Palavras-chave

- exercício físico

- monitores de aptidão física

- criança

- pesquisas e questionários

- tradução

- características culturais

- COVID-19

Objetivo Realizar a tradução e adaptação cultural para o português do Brasil do Children's Physical Activity Questionnaire.

Métodos $\mathrm{O}$ processo envolveu as etapas de tradução, síntese, retrotradução, e revisão pelo grupo de tradução. Foi então criada uma versão pré-final e, posteriormente, a versão final do questionário.

Resultados Devido ao estilo de vida da população, oito atividades descritas no questionário foram mudadas com o objetivo de adaptá-lo à cultura brasileira, sendo seis atividades esportivas e duas atividades de lazer. Oito pais/responsáveis responderam o questionário no pré-teste, com rapidez, sem inconsistências, e sem relatar dificuldades no entendimento quando realizada a entrevista final.

Conclusão A tradução e adaptação cultural do Children's Physical Activity Questionnaire para o português do Brasil resultou na versão C-PAQ.PT.
\end{abstract}

\footnotetext{
Trabalho desenvolvido no Programa de Pós-graduação em Ciências da Saúde Aplicada ao Esporte e à Atividade Física, Departamento de Ortopedia e Traumatologia, Escola Paulista de Medicina, Universidade Federal de São Paulo (Unifesp), São Paulo, SP, Brasil.
}

\section{Introdução}

Acelerômetros são instrumentos que atuam como sensores de movimento e permitem avaliar o nível e a frequência da recebido

24 de Fevereiro de 2021

aceito

15 de Junho de 2021
DOI https://doi.org/ 10.1055/s-0041-1736414. ISSN 0102-3616.

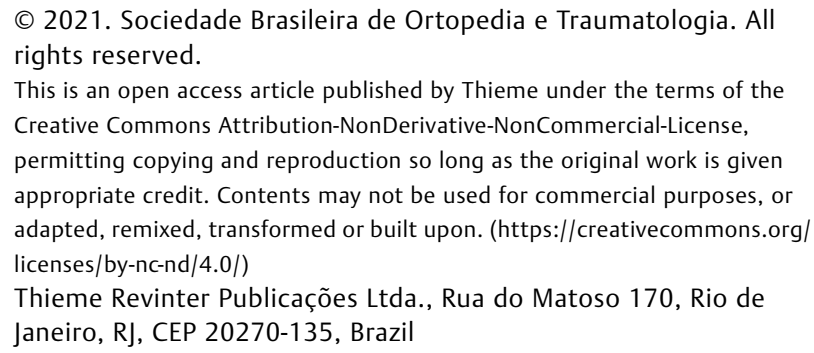

This is an open access article published by Thieme under the terms of the Creative Commons Attribution-NonDerivative-NonCommercial-License, permitting copying and reproduction so long as the original work is given appropriate credit. Contents may not be used for commercial purposes, or adapted, remixed, transformed or built upon. (https://creativecommons.org/ licenses/by-nc-nd/4.0/) Thieme Revinter Publicações Ltda., Rua do Matoso 170, Rio de Janeiro, RJ, CEP 20270-135, Brazil 


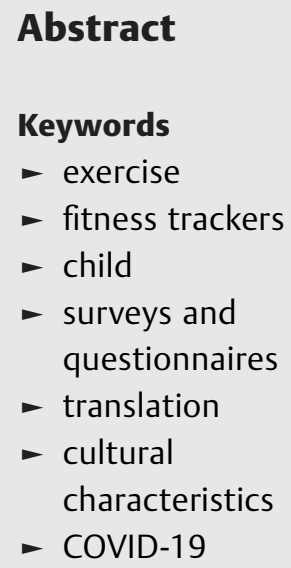

Objective To perform the translation and cultural adaptation to Brazilian Portuguese of the Children's Physical Activity Questionnaire

Methods The process involved the stages of translation, synthesis, back-translation, and revision by the translation group. A prefinal version of the questionnaire was then created, and, subsequently, the final version.

Results Due to the lifestyle of the population, eight activities described in the questionnaire were changed to adapt it to Brazilian culture: six sports activities and two leisure activities. Eight parents/legal guardians answered the questionnaire during the pretest, quickly, without inconsistencies, and without reporting difficulties in understanding when the final interview was conducted.

Conclusion The translation and cultural adaptation of the Children's Physical Activity Questionnaire to Brazilian Portuguese resulted in the version called C-PAQ.PT. atividade física em crianças. Seu uso permite coletar informações de maneira imediata, com baixo custo e grande aplicabilidade, levando em consideração diferentes contextos e a prática de atividade física. ${ }^{1,2}$ Em situações de isolamento social, como durante a pandemia de doença do coronavírus 2019 (coronavirus disease 2019, COVID-19, em inglês), a utilização de acelerômetro para esse tipo de pesquisa se torna praticamente impossível. Por esse motivo, buscamos na literatura outras formas que permitissem a avaliação do nível e da frequência de atividade física em crianças, sem comprometer o isolamento social.

Os questionários de autorrelato da rotina de atividade física são utilizados em larga escala como opção para o monitoramento de crianças. Existem diversos questionários, para diferentes faixas etárias, e, em sua maioria, validados pela comparação com o uso concomitante de acelerômetros. $^{3-6}$ O Children's Physical Activity Questionnaire (C-PAQ) é uma ferramenta que pode ser acessada livremente e baixada no site do Medical Research Council Epidemiology Unit/University of Cambridge School of Clinical Medicine (https://www.mrc-epid.cam.ac.uk/wp-content/uploads/ 2014/08/CPAQ.pdf). O questionário original em inglês teve validação prévia, ${ }^{7}$ e foi empregado em um estudo anterior. ${ }^{8}$ Em nossa opinião, o C-PAQ apresenta vantagens em relação aos demais questionários devido ao fato de ser constituído por perguntas sobre diferentes atividades comumente praticadas por crianças, como atividades esportivas, de lazer, as praticadas na escola, e atividades sedentárias. Devido a essas características, consideramos essa ferramenta ideal para avaliar o impacto do isolamento social consequente à pandemia de COVID-19 nas atividades de crianças e adolescentes residentes no Brasil. A tradução e adaptação cultural de questionários de autoavaliação requerem pouco tempo quando comparadas ao tempo necessário para a elaboração de um novo questionário. Essa metodologia permite que instrumentos que são bastante úteis na prática clínica sejam utilizados para comparar grupos de diferentes idiomas e culturas. ${ }^{9-13}$

O objetivo principal do presente estudo foi realizar a tradução e a validação cultural do C-PAQ para o português do Brasil.

\section{Materiais e Métodos}

Este estudo foi enviado ao comitê de ética em pesquisa pela nossa universidade, sendo aprovado em maio de 2020. Todos os pais/responsáveis assinaram eletronicamente o termo de consentimento.

\section{Children's Physical Activity Questionnaire (C-PAQ)}

Inicialmente, o questionário apresenta espaços a serem preenchidos com dados para a identificação da criança, como nome, data de nascimento, e a relação familiar do responsável que irá preencher o questionário. Em seguida, há uma breve explicação e orientações quanto ao preenchimento do questionário. O C-PAQ é constituído de perguntas referentes à prática de diferentes atividades cotidianas pela criança, incluindo atividades esportivas, de lazer, as praticadas na escola, e atividades sedentárias. Os pais ou responsáveis devem fornecer respostas levando em conta as atividades realizadas pela criança nos sete dias que precederam o preenchimento do questionário. Para facilitar o entendimento por parte dos pais/responsáveis, existe um exemplo com uma pergunta hipotética e com a forma correta de efetuar a resposta. As opções de respostas são simples: SIM ou NÃO. Em caso afirmativo, a intensidade é avaliada pela quantidade de horas que são despendidas na realização de cada atividade, tanto nos dias de semana quanto nos dias de finais de semana.

Os dados contidos no questionário preenchido permitem calcular o número de horas/minutos despendidos pela criança na realização de cada atividade no período de sete dias. ${ }^{7,8}$

\section{Processo de tradução e adaptação cultural}

Devido à sua importância na literatura, decidimos utilizar os trabalhos de Beaton et al. ${ }^{14}$ e Guillemin et al. ${ }^{15}$ como diretrizes para todo o processo de tradução e adaptação cultural. Essa metodologia obedece às etapas específicas necessárias de traduções iniciais e síntese das traduções, retrotradução, revisão por comitê de especialistas, e préteste até a versão final. 
Com a adaptação cultural, podemos considerar as diferenças culturais de percepção de saúde, idioma, contexto cultural, e estilo de vida da população em questão. Por esses métodos, é possível manter a equivalência linguística e cultural dos itens do instrumento, bem como sua relevância, mesmo com as modificações realizadas.

\section{Estágio 1}

O C-PAQ original foi inicialmente traduzido do inglês para o português do Brasil por dois tradutores, fluentes em ambos os idiomas, que criaram duas versões do questionário (B1 e B2).

\section{Estágio 2}

Os questionários B1 e B2 foram avaliados, comparados, e sintetizados por dois pesquisadores responsáveis pelo estudo, o que resultou no questionário B12.

\section{Estágio 3}

Foi elaborada nova tradução do questionário B12 para o inglês, também por dois tradutores fluentes em ambos os idiomas, sem conhecimento prévio da versão original, o que resultou nos questionários B12.1 e B12.2.

\section{Estágio 4}

Nesse momento, todos os documentos, as classificações originais, e as traduções (B1, B2, B12, B12.1 e B12.2) foram reavaliados por um comitê interdisciplinar. O comitê foi composto pelos pesquisadores, por um estudante de doutorado em economia fluente nos dois idiomas, uma fisioterapeuta, e um responsável por uma criança do estudo previamente selecionado com inglês fluente. Foram avaliadas todas as discrepâncias e interpretações equivocadas, e chegou-se a uma versão pré-final (B13).

\section{Estágio 5}

No pré-teste, a versão B13 foi aplicada a uma amostra da população-alvo, e, com base nas respostas, foram realizadas as alterações que o comitê julgou necessárias para melhorar o entendimento. $\mathrm{O}$ processo terminou com o questionário final.

\section{Resultados}

Devido ao estilo de vida da população em questão, as atividades contidas na versão original que são pouco realizadas no Brasil foram substituídas por atividades comumente realizadas, com o objetivo de adaptar o questionário à cultura brasileira. As trocas estão resumidas na - Tabela 1. As atividades esportivas do C-PAQ foram assim dispostas: aeróbica, base 4 , basquete/vôlei, queimada, dança, futebol, ginástica, handebol, lutas, rouba-bandeira, jogo de taco, corrida, aulas de natação, natação por diversão, e tênis. As de lazer foram as seguintes: andar de bicicleta (exceto à escola), empinar pipa, jogar boliche, realizar tarefas domésticas, brincar de casinha, brincar no parquinho, brincar com bichos de estimação, andar de patins, de patinete, de skate, brincar de polícia e ladrão, pular corda, brincar de pega-pega
Tabela 1 Resumo das mudanças de atividades devido ao estilo de vida da população brasileira

\begin{tabular}{|l|l|l|}
\hline & Original & $\begin{array}{l}\text { Trocas para a } \\
\text { adaptação cultural }\end{array}$ \\
\hline Atividade física & Baseball & Base 4 \\
\hline & Cricket & Jogo de taco \\
\hline & Martial arts & Lutas \\
\hline & Netball & Rouba-bandeira \\
\hline & Rugby & Handebol \\
\hline Lazer & Hockey & Queimada \\
\hline & $\begin{array}{l}\text { Bounce on the } \\
\text { trampoline }\end{array}$ & Empinar pipa \\
\hline & $\begin{array}{l}\text { Skiing, } \\
\text { snowboarding, } \\
\text { sledging }\end{array}$ & Polícia e ladrão \\
\hline
\end{tabular}

(todos os tipos), caminhar com o cachorro, e caminhada/trilha. As escolares foram assim dispostas: aula de Educação Física, ir para a escola a pé (ida e volta), e ir para a escola de bicicleta (ida e volta). E as sedentárias foram as seguintes: assistir a TV/vídeos, fazer arte e artesanato, fazer desenho e pintura, fazer a lição de casa, brincar de faz de conta, escutar música, brincar com brinquedos dentro de casa, jogar jogos de tabuleiro/cartas, jogar jogos de computador, brincar com eletrônicos, celular e tablet, tocar instrumento musical, leitura, sentar-se e conversar, falar ao telefone, ir e voltar para escola de carro ou ônibus, e navegar na internet ou outras atividades.

No pré-teste, o questionário foi enviado para oito pais, que o devolveram respondido. Todas as perguntas foram respondidas, e não observamos inconsistências nas respostas. Realizamos contato por telefone para entrevista com os pais, que relataram não ter tido dificuldade para responder às perguntas.

Após o último estágio, chegou-se à versão pré-final (B13) da tradução e adaptação cultural para o português do Brasil do C-PAQ. Denominamos essa versão final de C-PAQ.PT (-Apêndice 1, disponível na versão on-line).

\section{Discussão}

A pandemia de COVID-19 impactou a rotina de vida das crianças em todo o mundo. $\mathrm{O}$ isolamento social refletiu diretamente na intensidade e na frequência das atividades esportivas e de lazer. Na educação de crianças e jovens, é fundamental garantir uma rotina diária de hábitos de atividade física com o intuito de se manter uma vida saudável.

O C-PAQ permite uma adequada avaliação, de baixo custo, fácil aplicabilidade, e boa aceitação dos participantes devido à agilidade na coleta dos dados. ${ }^{7,8}$ Apesar de apresentar 49 perguntas, as respostas são simples, o que permite que o questionário seja respondido em aproximadamente dez minutos.

Durante o isolamento social provocado pela COVID-19, a diminuição da frequência e da intensidade da atividade física entre as crianças pode impactar na prática ortopédica. Raitio et al. $^{16}$ observaram uma diminuição significativa dos 
procedimentos cirúrgicos decorrentes das lesões relacionadas com as atividades diárias, escolares e esportivas das crianças durante o período de isolamento social. Em um centro de trauma italiano, ${ }^{17}$ o número de pacientes pediátricos diminuiu $84,6 \%$, não se observando no período nenhuma lesão relacionada a traumas na escola, de alta energia, ou traumas decorrentes da prática esportiva. No entanto, o sedentarismo em crianças está relacionado à obesidade, que, por sua vez está relacionada a complicações ortopédicas durante o crescimento e o desenvolvimento da criança. ${ }^{18}$

Os métodos de tradução e adaptação cultural são amplamente descritos e utilizados para a avaliação, o acompanhamento e a comparação de diferentes afecções ou situações na prática clínica da ortopedia e medicina esportiva. ${ }^{8,11,19} \mathrm{~A}$ adaptação cultural é realizada por meio de dois componentes básicos: a tradução do instrumento de seu idioma original para outro, e sua adaptação à cultura e ao estilo de vida da população que utilizará o questionário. ${ }^{14}$ Nesse processo, certas situações ou questões podem ser mudadas integralmente ou parcialmente. Essas mudanças, entretanto, devem manter as mesmas características do original para garantir a mesma mensuração. ${ }^{20}$ O C-PAQ original é objetivo, e apresenta perguntas e respostas curtas, que facilitaram o trabalho de tradução literal do inglês para o português do Brasil. Entretanto, nossa maior dificuldade foi decorrente do fato de haver algumas perguntas sobre atividades esportivas que não são praticadas por crianças no Brasil. Essa situação é descrita como equivalência de experiência, quando uma determinada atividade não pode ser realizada, mesmo que possa ser traduzida. Nessa situação, o termo deve ser substituído por um termo que designe algo que possa ser realizado pela população-alvo da tradução. ${ }^{20}$ Como preconizado por Reichenheim et al., ${ }^{21}$ promovemos discussões com especialistas e consultamos membros da população-alvo para realizar as trocas e aprovar a equivalência dos itens. Como descrito na -Tabela 1, as atividades pouco praticadas pelas crianças no Brasil foram trocadas por atividades praticadas em nosso meio, procurando manter as mesmas características de intensidade. Sendo assim, por exemplo, a atividade física skiing, snowboarding, sledging foi trocada por outra atividade física frequente, no caso, a brincadeira de polícia e ladrão, sem a necessidade de traduzir por uma atividade física que use uma prancha, esquis ou trenó. Devemos lembrar que o C-PAC avalia não só a intensidade da atividade física, como também atividades de lazer, as praticadas na escola, e as sedentárias. O que não poderíamos fazer seria trocar uma atividade física por uma não física. A formação de um comitê interdisciplinar no estágio 4 do processo tem o objetivo de evitar vieses de preferências dos autores na adaptação cultural, como a preferência por um tipo de atividade física, e, na tradução, para evitar palavras técnicas que dificultem o entendimento pelo público leigo.

O pré-teste compreende a aplicação do questionário a uma pequena população-alvo, de número mínimo recomendado que varia de dez a quarenta participantes. ${ }^{14-22}$ Em sua estrutura, o questionário é constituído por palavras do cotidiano de qualquer pessoa, que incluem os tipos de atividades, as respostas afirmativa e negativa ( im e não), e medidas temporais, como minutos, horas e dias da semana. Essa característica do questionário impede que tenhamos diferentes respostas individuais ou pontuações mais baixas ou mais altas. Em nossa pesquisa, o pré-teste foi aplicado apenas a oito participantes; consideramos esse número suficiente devido à rapidez com que recebemos as respostas, à consistência dessas respostas, $\mathrm{e}$ ao resultado da entrevista com os pais/responsáveis, que relataram seu fácil entendimento e preenchimento. Para a avaliação da versão final, o C-PAQ.PT, iniciamos uma pesquisa para avaliar o impacto do isolamento social nas atividades de crianças e adolescentes; os dados de 136 questionários estão sendo copilados, e serão tema de estudo.

Como o Brasil é um país de grande extensão territorial, com algumas diferenças culturais internas, acreditamos que algumas trocas não afetam a estrutura do questionário, que apresenta um grande número de opções de atividades físicas.

\section{Conclusão}

Consideramos o C-PAQ traduzido e validado culturalmente para o português do Brasil, que denominamos C-PAQ.PT.

\section{Fontes de Financiamento}

Esta pesquisa não recebeu nenhuma bolsa específica de agências de fomento nos setores público, comercial, ou sem fins lucrativos.

Conflito de Interesses

Os autores declaram não haver conflito de interesses.

\section{Referências}

1 Bender JM, Brownson RC, Elliott MB, Haire-Joshu DL. Children's physical activity: using accelerometers to validate a parent proxy record. Med Sci Sports Exerc 2005;37(08):1409-1413

2 Cain KL, Sallis JF, Conway TL, Van Dyck D, Calhoon L. Using accelerometers in youth physical activity studies: a review of methods. J Phys Act Health 2013;10(03):437-450

3 Basterfield L, Adamson AJ, Parkinson KN, et al. Surveillance of physical activity in the UK is flawed: validation of the Health Survey for England Physical Activity Questionnaire. Arch Dis Child 2008;93(12):1054-1058

4 Burdette HL, Whitaker RC, Daniels SR. Parental report of outdoor playtime as a measure of physical activity in preschool-aged children. Arch Pediatr Adolesc Med 2004;158(04):353-357

5 Haskell WL. Physical activity by self-report: a brief history and future issues. J Phys Act Health 2012;9(Suppl 1):S5-S10

6 Welk GJ, Corbin CB, Dale D. Measurement issues in the assessment of physical activity in children. Res Q Exerc Sport 2000;71(2 Suppl)S59-S73

7 Corder K, van Sluijs EM, Wright A, Whincup P, Wareham NJ, Ekelund U. Is it possible to assess free-living physical activity and energy expenditure in young people by self-report? Am J Clin Nutr 2009;89(03):862-870

8 Anderson YC, Wynter LE, Grant CC, et al. Physical activity is low in obese New Zealand children and adolescents. Sci Rep 2017; 7:41822

9 Ferreira MC, Silva G, Zidan FF, Franciozi CE, Luzo MVM, Abdalla RJ. Tradução e adaptação cultural para a língua portuguesa do instrumento de avaliação para artroplastias de quadril e joelho Forgotten Joint Score. Rev Bras Ortop 2018;53(02):221-225

10 Almeida VAS, Fernandes CH, Meirelles LM, Santos JBG, Faloppa F, Ejnisman B. Translation and Cross-cultural Adaptation of the "Thumb Disability Exam - TDX" questionnaire into Brazilian 
Portuguese [Published online: 2020-09-22]. Rev Bras Ortop. Available from: https://www.thieme-connect.com/products/ejournals/html/10.1055/s-0040-1715508

11 Lenzi L, Santos J, Raduan Neto J, Fernandes CH, Faloppa F. The Patient and Observer Scar Assessment Scale: Translation for portuguese language, cultural adaptation, and validation. Int Wound J 2019;16(06):1513-1520

12 Matsuo RP, Fernandes CH, Meirelles LM, Raduan Neto J, Dos Santos JB, Fallopa F. Translation and Cross-Cultural Adaptation of the 6Item Carpal Tunnel Syndrome Symptoms Scale and Palmar Pain Scale Questionnaire Into Brazilian Portuguese. Hand (N Y) 2016; 11(02):168-172

13 Fernandes CH, Neto JR, Meirelles LM, Pereira CN, Dos Santos JB, Faloppa F. Translation and cultural adaptation of the Brief Michigan Hand Questionnaire to Brazilian Portuguese language. Hand (N Y) 2014;9(03):370-374

14 Beaton DE, Bombardier C, Guillemin F, Ferraz MB. Guidelines for the process of cross-cultural adaptation of self-report measures. Spine 2000;25(24):3186-3191

15 Guillemin F, Bombardier C, Beaton D. Cross-cultural adaptation of health-related quality of life measures: literature review and proposed guidelines. J Clin Epidemiol 1993;46(12):1417-1432

16 Raitio A, Ahonen M, Jääskelä M, et al. Reduced Number of Pediatric Orthopedic Trauma Requiring Operative Treatment during
COVID-19 Restrictions: A Nationwide Cohort Study. . [published online ahead of print, 2020 Oct 26]Scand J Surg 2020; 110(02): 254-257

17 Gumina S, Proietti R, Villani C, Carbone S, Candela V. The impact of COVID-19 on shoulder and elbow trauma in a skeletally immature population: an Italian survey. JSES Int 2021;5(01):3-8

18 Taylor ED, Theim KR, Mirch MC, et al. Orthopedic complications of overweight in children and adolescents. Pediatrics 2006;117(06): 2167-2174

19 Silva ALP, Croci AT, Gobbi RG, Hinckel BB, Pecora JR, Derange MK. Translation and validation of the new version of the Knee Society Score - The 2011 KS Score - into Brazilian Portuguese. Rev Bras Ortop 2017;52(04):506-510

20 Dortas Junior SD, Lupi O, Dias GA, Guimarães MB, Valle SO. Adaptação transcultural e validação de questionários na área da saúde. Braz J Allergy Immunol 2016;4(01):26-30

21 Reichenheim ME, Moraes CL. Operacionalização de adaptação transcultural de instrumentos de aferição usados em epidemiologia. Rev Saude Publica 2007;41(04):665-673

22 Baiardini I, Bousquet PJ, Brzoza Z, et al. Recommendations for assessing patient-reported outcomes and health-related quality of life in clinical trials on allergy: a GA(2) LEN taskforce position paper. Allergy 2010;65(03):290-295 